\title{
Interactive EBSD Based Analysis of a Fatigue Crack in a Nickel Superalloy
}

\author{
Matthew M. Nowell,* Stuart I. Wright,* and Rene de Kloe** \\ * EDAX-TSL, 392 E 12300 S, Suite H, Draper, UT 84020 \\ ** EDAX BV, Ringbaan Noord 103, P.O.Box 4144, 5004 JC Tilburg, The Netherlands
}

The automation of the EBSD technique has enabled the practical analysis of the orientation aspects of polycrystalline microstructures. The various commercial systems provide an array of tools for the statistical analysis of the EBSD data. Despite these advances, it is sometimes necessary for a researcher to manually investigate the data in order to discover the most critical features of the microstructure. For example, cracks in maps constructed from EBSD data can be recognized due to the lack of data obtained from cracked regions. Thus, the cracks may appear as dark regions in maps based on the image quality of the diffraction patterns or the lack of color in color maps based on orientation. However, because cracks may mimic grain boundaries in these maps, it is difficult to accurately extract the crack location from the EBSD data. Thus, in order to elucidate the influence of crystallographic orientation on the propagation of the crack through the microstructure both in terms of intergranular and transgranular propagation, the researcher must manually interrogate the data. This work reports on such analysis of a nickel superalloy [1].

This work shows an example of interactive analysis of a fatigue crack. The orientation data was correlated with the trace of the crack through the microstructure to identify the interaction of crystallographic planes with the propagation of the crack. The study found that the crack preferentially propagates along $\{111\}$ planes in the microstructure both when propagating along boundary planes as well as when propagating through the grains. It was noted that when the crack traveled transgranularly, that it would be much more jagged than when propagating intergranularly. The jags are formed as the crack alternates between different $\{111\}$ planes in the crystal. This conclusion was reached by analysis of the $\{111\}$ traces near the edge of the crack as shown in figure 1 .

A high resolution EBSD scan was performed near the apparent leading tip of the crack (it is possible the crack dipped below the surface so that the leading tip of the crack cannot be observed in the scanned section plane). Whenever, the crack jumps to a new $\{111\}$ plane, a region of high local misorientation is observed in the EBSD data indicating the presence of high dislocation density as shown in figure 2. However, it is not apparent whether the dislocations build up at the tip of the crack causing it to switch planes or if they are a residual of the change in crack direction. One feature observed in the local misorientation data is that the dislocations seem to cause a rotation of the region near the crack to rotate about an axis normal to the specific (111) plane along which the crack is propagating.

References

[1] D.L. Davidson et al., Metall. Mater. Trans., 38 (13) (2007) 2214.

[2] R. Chandran of the University of Utah is acknowledged for supplying the fatigued samples. 


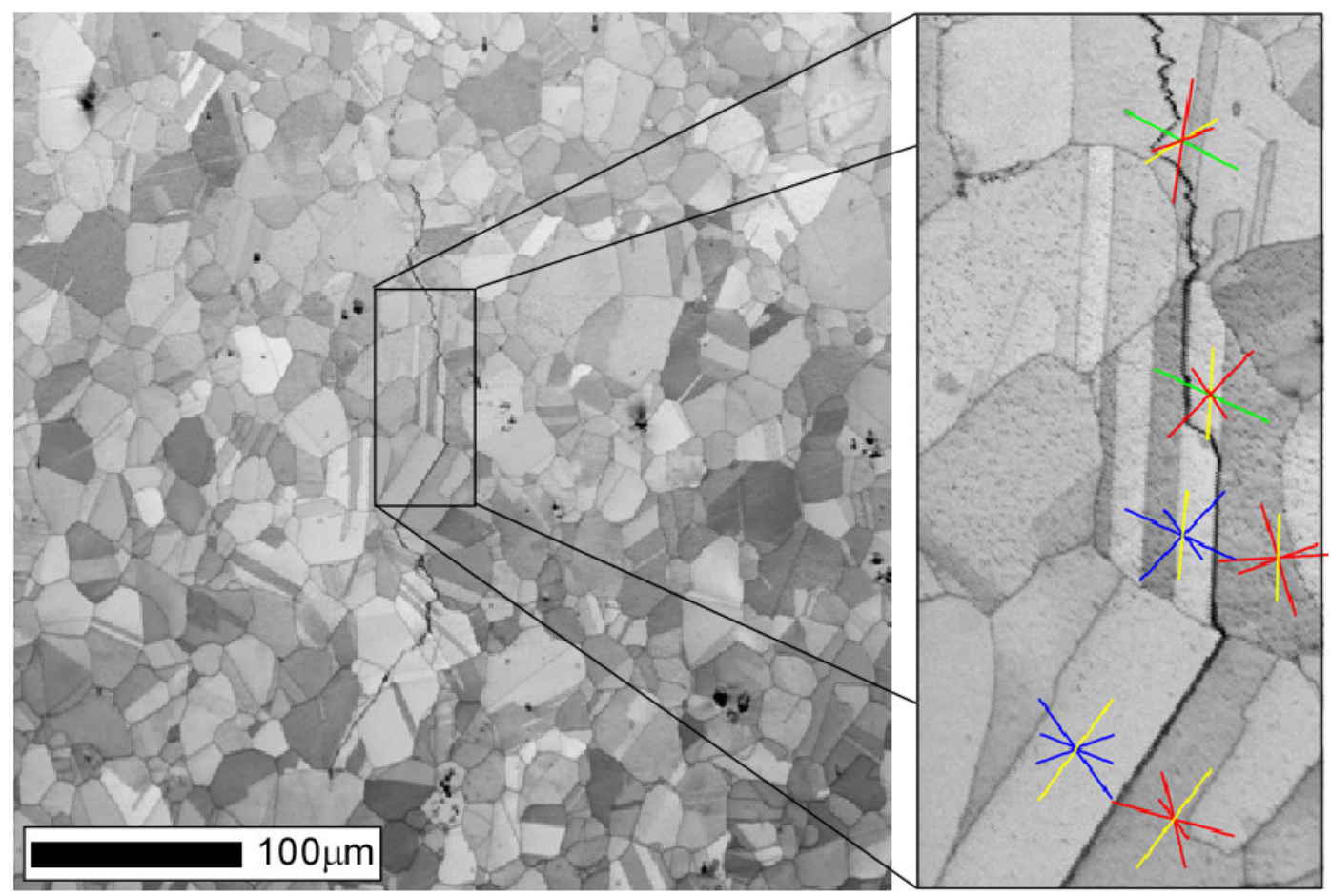

FIG. 1. Image quality map from EBSD data on a fatigued nickel superalloy and traces of $\{111\}$ planes in grains neighboring the crack. The traces highlighted in yellow and green are those parallel to the trace of the crack.

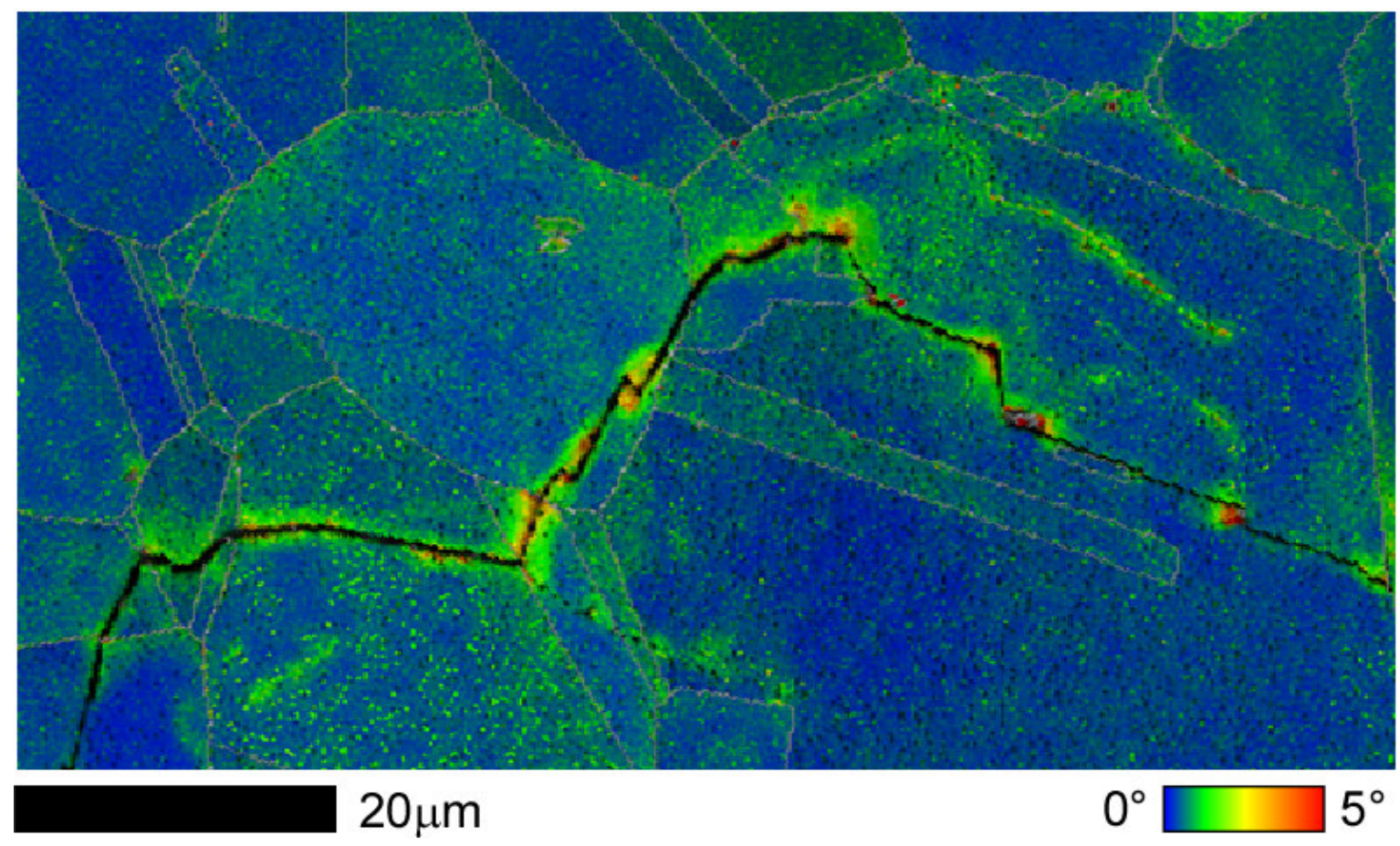

FIG. 2. Local misorientation map for a fatigue crack in a nickel superalloy. Note the red color indicating high local misorientation near the kinks in the crack. 